\title{
The impact of a pharmacist-led educational interview on medication adherence of Saudi patients with epilepsy
}

\author{
Refah AIAjmi' \\ Sinaa Al-Aqeel' \\ Salah $\mathrm{Baz}^{2}$ \\ 'Clinical Pharmacy Department, \\ College of Pharmacy, King Saud \\ University, Riyadh, Kingdom of \\ Saudi Arabia; ${ }^{2}$ Department of \\ Neurosciences, King Faisal Specialist \\ Hospital and Research Center, Riyadh, \\ Kingdom of Saudi Arabia
}

This article was published in the following Dove Press journal:

Patient Preference and Adherence

18 May 2017

Number of times this article has been viewed

Purpose: To evaluate the effectiveness of a pharmacist-led educational interview in terms of adherence to antiepileptic drug administration among adult patients with epilepsy.

Method: Sixty adult patients with epilepsy who fulfilled the inclusion criteria were recruited. A pharmacist-led educational interview was conducted with the intervention group $(n=30)$. Patients in the control group $(n=30)$ were interviewed and contacted 6 weeks after the initial visit without receiving any intervention. Antiepileptic drug adherence was measured during clinic visits, and 6 weeks afterwards using the 8-item Morisky Medication Adherence Scale. This prospective interventional study was conducted between September and December 2013.

Results: Only 29 control patients and 27 intervention patients completed the 6 weeks post-intervention adherence measurement. The adherence score average in the intervention group was $5.26 \pm 0.98$ at baseline and improved to $6.7 \pm 0.823(P<0.0001)$ after intervention. In the control group, the adherence score average was $5.76 \pm 1.806$ at baseline and $5.83 \pm 1.627$ at 6 weeks $(P=0.792)$. While there was no statistically significant difference in adherence score between intervention and control groups at baseline, the post-intervention difference was significant $(P=0.024)$.

Conclusion: Our study suggests that pharmacist-led educational interviews had a positive impact on medication adherence in patients with epilepsy.

Keywords: epilepsy, antiepileptic drugs, adherence

\section{Introduction}

Epilepsy is a neurological disorder characterized by an enduring predisposition to epileptic seizures as well as the neurobiological, cognitive, psychological, and social consequences of this condition. ${ }^{1}$ Epilepsy is one of the most common neurological disorders worldwide with median incidence of 50.4 per 100,000 people per year (interquartile range [IQR]: 33.6-75.6). The median incidence for high-income countries is 45.0 per 100,000 people per year (IQR: 30.3-66.7) and 81.7 (IQR: 28.0-239.5) per 100,000 people per year for low- and middle-income countries. ${ }^{2}$ In the Kingdom of Saudi Arabia, the prevalence is 6.54 per 1,000 people per year. ${ }^{3}$

The World Health Organization defines medication adherence as the extent to which a patient's behavior, in terms of taking medications, is in agreement with their provider's recommendations. ${ }^{4}$ A consensus group ${ }^{5}$ described the process of medication adherence as initiation (ie, the patient takes the first dose of a prescribed medication), implementation (ie, the extent to which the patient's actual dosing corresponds to the prescribed dosing regimen), and persistence (ie, the length of time on regimen between initiation and the last dose before discontinuation). Thus, non-adherence to
Correspondence: Sinaa Al-Aqeel

Clinical Pharmacy Department, College

of Pharmacy, King Saud University,

PO Box 3763 I6, Riyadh II335,

Kingdom of Saudi Arabia

Email salageel@ksu.edu.sa 
medication can occur if a patient failed to initiate treatment, sub-optimally implemented the dosing regimen, and/or discontinued the treatment early.

Adherence is determined by many factors that can be grouped under five dimensions: social and economical, health system related, therapy related, condition related, and patient related. ${ }^{4}$ Determinants of adherence to antiepileptic drug (AED) regimens include the complexity of the drug regime, uncontrolled seizure, patients' understanding about their illness, age, education level, and employment. ${ }^{6-8}$

Periods of adherence failure to AED regimens are associated with increased risk of seizure, status epilepticus, and mortality. There is a significantly higher incidence of emergency department visits, hospital admissions, injuries, and fractures than during periods of adherence - this results in higher resource utilization and costs..$^{9-11}$

A recent review on strategies to improve adherence to antiepileptic medications identified three main types of interventions: educational, behavioral, and mixed. ${ }^{12}$ The assumption for educational interventions is that patients who understand their illness and its treatment will be more informed, have more control, and be more likely to adhere to treatment. ${ }^{13}$ This is particularly important given the evidence of lack of awareness about the medical condition and indications for AEDs among patients with epilepsy, and their needs for information and counseling. ${ }^{14,15}$ However, published evidence on the effectiveness of this approach reported mixed results. ${ }^{12}$

Previous evidence suggests that pharmacists with knowledge about pharmacotherapy and management of chronic diseases, and training in health education could contribute positively toward adherence to antidepressant and antidiabetic medications. ${ }^{16,17}$ A systematic review of published studies describing clinical services performed by pharmacists for patients with epilepsy, identified five studies that highlighted the positive impact of pharmacists' interventions on knowledge, quality of life, and medication adherence in this group of patients. ${ }^{18}$

Considering the inconclusive evidence on the efficacy of educational interventions and the paucity of studies about pharmacists' contributions to epilepsy management, this study evaluates the effectiveness of a pharmacist-led educational interview in terms of adherence to AED administration among adult patients with epilepsy.

\section{Materials and methods Participants}

The study was conducted at the outpatient Epilepsy Clinic at King Faisal Specialist Hospital and Research Center (KFSH and RC) - a tertiary referral hospital in Riyadh, Kingdom of Saudi Arabia. The inclusion criteria for patients were a clinical diagnosis of epilepsy, age $\geq 18$ years, current use of AEDs, and the ability to provide consent and complete the scale independently. Patients were excluded if they had any mental problems that impaired their ability to complete the scale or if they refused to participate. Patients who met the inclusion criteria were invited to participate in the study by one of the investigators (SB). Patients were given a participation information sheet that described the type of study, and the nature and purpose of the intervention. Those who agreed to participate signed the consent form. The Office of Research Affairs and Ethics Committee of KFSH and RC approved this study. Patients recruited in September 2013 were allocated to the control group, while those recruited in October through December 2013 were allocated to the intervention group. Participants did not receive incentives.

\section{Intervention}

This study was designed as a prospective nonrandomized interventional study. The educational interview involved a 30-minute structured verbal face-to-face interview conducted by one of the researchers (RA) who is a pharmacist. All patients were educated according to the guidelines of the American Society of Health-System Pharmacists regarding pharmacist-conducted patient education and counseling. ${ }^{19}$ Patients in the intervention group were interviewed individually and privately, either in the clinic after the consultant's examination or in the nurse's room.

The educational content was divided into two parts. The first part included education about the medical aspects of epilepsy, and the second part included information about AEDs. Leaflets that contained the content of the educational interview were written in Arabic and were presented in a "question and answer" format, and given to the intervention group. This leaflet included educational information about the medical aspects of epilepsy including the definition of epilepsy, causes, description of brain seizure activity, diagnostic procedures, seizure observation, and classification; information about AEDs; and information about the importance of adherence and the consequences of non-adherence. The pharmacists also discussed methods to incorporate AED administration into their daily lives and to transform their daily routines into cues to take their AEDs. The most common cue was prayer times. In addition, the pharmacist provided a pill organizer to each participant and counseling about how to use it, and its usefulness.

For more assistance and support, participants were advised to visit an Epilepsy Support and Information Center (ESIC) - a non-profit regional office governed by KFSH 
and RC, under the "umbrella" of the International Bureau of Epilepsy, that provides information and support to epilepsy patients and their families. The leaflet also provided the web address for ESIC for patients to access online information. All participants received the usual care offered by their clinic. Patients in the control group did not receive an educational interview from the pharmacist, a pill organizer, or leaflets. They could access the ESIC, but the pharmacist did not encourage it.

\section{Study measures}

The instrument used here consisted of a 2-part questionnaire. Part one included demographic data, and part two included medication adherence questions. Demographic characteristics included age, gender, marital status, occupational status, education, age of onset of epilepsy, seizure frequency, the time since the last seizure, and the number of AEDs taken. In this study, self-reported adherence was assessed using the 8-item Morisky Medication Adherence Scale (MMAS-8). ${ }^{20-22}$ The scale provides information on behavior related to low adherence that may be unintentional (eg, forgetfulness) or intentional (eg, stopped taking medications because they felt that their symptoms were under control). The scale contains items that seek to assess the extent of implementation of a dosing regimen and discontinuation of treatment.

The MMAS- 8 has been used previously to assess medication adherence in epilepsy patients. ${ }^{23-25}$ The license to use the validated Arabic version of the MMAS- 8 was obtained. The version is in modern standard Arabic language - the language used for reading and writing in Arab countries including Kingdom of Saudi Arabia. The MMAS is an 8 -item questionnaire with seven items represented in a yes/no format. The final question is rated on a 5-point Likert scale. According to the MMAS scoring system, a score of 8 reflects high adherence, and 6 to $<8$ reflect medium adherence; scores $<6$ reflect low adherence. ${ }^{20}$

The questionnaire was administered to both the intervention and control groups at baseline as well as 6 weeks after the intervention. While baseline measurements were carried out at the clinic, adherence levels post-intervention were assessed via a phone call to both groups.

\section{Statistical analysis}

A pilot study indicated that a sample size of 27 subjects in each group was sufficient. The size was estimated based on an average MMAS of 5.2 \pm 1.6 with an expected adherence difference of within $24 \%$ at a significance level of 5\%.

Descriptive analyses including the means and standard deviations (SDs) were calculated for all quantitative values.
Differences between the group's adherence levels were compared using a Wilcoxon signed rank test. The mean, median, and interquartile ranges were used for differences between adherence scores. Spearman's correlation coefficient was used to assess the relationship between age, education level, duration of illness, number of seizure episodes, or number of AEDs and adherence score. Chi-square tests, Mann-Whitney $U$ tests, and independent $t$-tests were used to test for differences in patients' characteristics such as seizure frequency, as appropriate. The statistician was blinded to which group received the intervention. Analysis was conducted to determine the effect of intervention after 6 weeks. Statistical analysis was carried out using the Statistical Package for the Social Sciences (SPSS) version 19 software (IBM Corporation, Armonk, NY, USA).

\section{Results}

Seventy patients were approached, and 60 agreed to participate; 30 were assigned to each group. Only 29 control patients and 27 intervention patients completed the 6 weeks post-intervention adherence measurement. The loss to follow-up was due to failure to reach the patients through a post-intervention phone call.

\section{Participant characteristics}

The participant characteristics are summarized in Table 1. The majority of participants were men aged $<40$ years, with a diagnosis of epilepsy for $\geq 10$ years. The mean $( \pm S D)$ number of AEDs were $2.34( \pm 0.93)$ and $2.03( \pm 1.05)$ for control and intervention groups, respectively. The average number of seizure episodes during the last 6 months ranged between $4.96( \pm 5.29)$ and $4.72( \pm 3.26)$ for the intervention and control groups, respectively. There were no statistically significant differences in age, gender, educational status, marital status, or seizure frequencies between the intervention and control groups.

Only 12 participants (44.0\%) used the pill organizer and incorporated it into their medication-taking behavior.

\section{Adherence}

The adherence score average in the intervention group was $5.26 \pm 0.98$ at baseline, and this improved to $6.7 \pm 0.823$ $(P<0.0001)$ after intervention. In the control group, the adherence score average was 5.76 \pm 1.806 at baseline and $5.83 \pm 1.627$ at 6 weeks $(P=0.792)$. While there was no statistically significant difference in adherence scores between intervention and control groups at baseline, the postintervention difference was significant $(P=0.024)$. There was no statistically significant correlation between medication 
Table I Demographics and clinical characteristics of the study population

\begin{tabular}{|c|c|c|}
\hline Characteristic & $\begin{array}{l}\text { Control } \\
\text { group N (\%) }\end{array}$ & $\begin{array}{l}\text { Intervention } \\
\text { group N (\%) }\end{array}$ \\
\hline \multicolumn{3}{|l|}{ Gender } \\
\hline Male & $16(55.2)$ & $14(5 \mid .9)$ \\
\hline Female & $13(44.8)$ & $13(48.1)$ \\
\hline \multicolumn{3}{|l|}{ Age (years) } \\
\hline $20-30$ & $12(4 \mid .4)$ & $15(55.6)$ \\
\hline $31-40$ & $12(4 \mid .4)$ & $6(22.2)$ \\
\hline $4 I-50$ & $3(10.3)$ & $5(18.5)$ \\
\hline $5 I-60$ & $2(6.9)$ & I (3.7) \\
\hline \multicolumn{3}{|l|}{ Marital status } \\
\hline Single & I4 (48.3) & I5 (55.6) \\
\hline Married & $14(48.3)$ & $12(44.4)$ \\
\hline Widowed & I (3.4) & $0(0)$ \\
\hline \multicolumn{3}{|l|}{ Education level } \\
\hline Primary & $7(24.1)$ & $4(143.8)$ \\
\hline Intermediate & $5(17.2)$ & $3(11.1)$ \\
\hline High school & $10(34.5)$ & $12(44.4)$ \\
\hline University & $7(24.1)$ & $8(29.6)$ \\
\hline \multicolumn{3}{|l|}{ Live with family } \\
\hline Yes & $24(82.8)$ & 21 (77.8) \\
\hline No & $5(17.2)$ & $6(22.2)$ \\
\hline \multicolumn{3}{|l|}{ Occupational status } \\
\hline Working & II (37.9) & $9(33.3)$ \\
\hline Not working & $18(62.1)$ & $18(66.7)$ \\
\hline \multicolumn{3}{|l|}{ Duration of illness (years) } \\
\hline$<10$ & $9(31)$ & II (40.7) \\
\hline$\geq 10$ & $20(69)$ & $16(59.3)$ \\
\hline \multicolumn{3}{|l|}{ Seizure frequency } \\
\hline Daily & $7(24.1)$ & $5(18.5)$ \\
\hline Last seizure was within a month & $13(44.8)$ & $7(25.9)$ \\
\hline Last seizure was within 3 months & $6(20.7)$ & $5(18.5)$ \\
\hline \multicolumn{3}{|l|}{ Number of AEDs } \\
\hline 1 & $6(20.7)$ & $8(29.6)$ \\
\hline$>1$ & $23(79.3)$ & $19(70.4)$ \\
\hline \multicolumn{3}{|l|}{ Type of AEDs } \\
\hline Carbamazepine & $16(55.2)$ & $17(63.0)$ \\
\hline Levetiracetam & $18(62.1)$ & $14(5 \mid .9)$ \\
\hline Lamotrigine & $10(34.5)$ & $8(29.6)$ \\
\hline Topiramate & $14(48.3)$ & $7(25.9)$ \\
\hline Valproic acid & $7(24.1)$ & $8(29.6)$ \\
\hline Phenytoin & $3(10.3)$ & I (3.7) \\
\hline
\end{tabular}

Abbreviation: AEDs, antiepileptic drugs.

adherence scores and age, education level, duration of illness, number of seizure episodes, or number of AEDs.

Table 2 shows the distribution of patients based on adherence level. At baseline, $40.7 \%$ of patients in the intervention group had medium adherence scores (MMAS-8 score, 6-8), and none were classified as having high adherence scores (MMAS-8 score, 8). Six weeks after intervention, 85.2\% of patients in the intervention group had medium adherence scores (MMAS- 8 score, 6 to $<8$ ) and $11.1 \%$ had high adherence scores (MMAS-8, score 8).

\section{Discussion}

The aim of this study was to investigate the effectiveness of pharmacist-led educational interview in epilepsy patients' adherence to AED regimens. The adherence score improved significantly in the intervention group patients.

Compared with previous studies that used a MMAS- 8 to measure medication adherence in patients with epilepsy, ${ }^{7,23,25}$ our participants had higher rates of moderateto-low adherence (MMAS-8 score, <8). Two-thirds of our participants were on polytherapy, which is higher than rates reported in other studies. ${ }^{23,25}$ This is probably attributable to the nature of the clinic from which the patients were recruited (an epilepsy clinic in a tertiary referral center).

Our results suggest that educational interview could positively impact the medication adherence of patients with epilepsy. Previous evidence on the effectiveness of educational interventions in terms of medication adherence in patients with epilepsy is mixed. ${ }^{12} \mathrm{Li}$ et $\mathrm{al}^{26}$ found that self-reported adherence remarkably improved in participants who received intensive education, consultation services, and repeated reminders, while unchanged adherence levels were reported in the non-intervention cohort. Peterson et $\mathrm{al}^{27}$ reported a positive impact on patient adherence to AEDs among those who received counseling, a pill organizer, and other reminder techniques, measured using blood tests and refill frequencies.

In another study, there was no significant difference in adherence to AEDs based on detectable drug levels or by self-reporting between the intervention and control groups, 1 year after a day-long health educational program..$^{28}$ Dash et $\mathrm{al}^{23}$ investigated the effect of a structured one-on-one educational program administered by an epilepsy nurse over four sessions each lasting at least 30 minutes. They found that self-reported adherence scores were significantly improved in the intervention group with no significant change in the control group.

The current study's findings suggest that pharmacists can improve the utilization of treatment by educating patients with epilepsy. Similar research, albeit limited in number, shows similar directions. A randomized controlled trial comparing AEDs education provided by a pharmacist with AEDs plus behavioral intervention, found that adherence increased greatly from baseline in both groups, but without significant differences. ${ }^{29}$ In a before/ after study conducted in adults with epilepsy, ${ }^{30}$ a single 30-minute consultation with a pharmacist was associated with improved self-reported adherence 2 months after the intervention. 
Table 2 Comparison of adherence level between control $(N=29)$ and intervention $(N=27)$ groups

\begin{tabular}{|c|c|c|c|c|c|c|}
\hline \multirow{2}{*}{$\begin{array}{l}\text { Adherence } \\
\text { level (points) }\end{array}$} & \multicolumn{2}{|l|}{ Baseline, n (\%) } & \multirow[t]{2}{*}{$P$-value } & \multicolumn{2}{|c|}{6 weeks after intervention, $n(\%)$} & \multirow[t]{2}{*}{$P$-value } \\
\hline & Control group & Intervention group & & Control group & Intervention group & \\
\hline $\begin{array}{l}\text { High } \\
\text { (8) }\end{array}$ & $7(24.1)$ & $0(0)$ & $0.007 * *$ & $4(13.8)$ & $3(11.1)$ & $0.54 I^{* *}$ \\
\hline $\begin{array}{l}\text { Medium } \\
(6-8)\end{array}$ & II (37.9) & II (40.7) & $0.830 *$ & $14(48.3)$ & $23(85.2)$ & $0.004 *$ \\
\hline $\begin{array}{l}\text { Low } \\
(<6)\end{array}$ & II (37.9) & $16(59.3)$ & $0.110 *$ & II (37.9) & I (3.7) & $0.002^{*}$ \\
\hline
\end{tabular}

Notes: *By Chi-square test; **by Fisher's exact test.

We did not assess the feasibility and costs of implementing such an intervention into pharmacists' routine care for patients with epilepsy. Published evidence suggests that adherence-enhancing interventions are cost-effective or costsaving. ${ }^{31,32}$ However, more studies on the cost-effectiveness of pharmacist-delivered interventions to improve adherence are needed.

Although our study found a statistically significant difference in adherence score between baseline and 6 weeks after the educational interview, the clinical impact remains unclear. The level at which non-adherence becomes clinically significant varies between medications, and the needed adherence rate is unknown for many medications. ${ }^{33}$ Research examining the level at which nonadherence rates to AEDs becomes clinically significant is needed.

This study is one of the very few studies ${ }^{29,30}$ that has examined the efficacy of pharmacist-led educational interview in patients with epilepsy. It does have some obvious limitations. The study was a prospective interventional study with no randomization. This might introduce selection bias. The majority of participants have had a clinical diagnosis of epilepsy for $>10$ years; thus, we cannot generalize our findings to newly diagnosed patients with epilepsy. The difference in collection periods between control and intervention groups is also a weakness. The fact that less than half of the participants used the pill organizer, limited our ability to assess the impact of this simple intervention. Previous studies have shown that reminder packaging has a positive impact on medication adherence. ${ }^{34} \mathrm{We}$ also used a self-reported adherence measure. Self-reported measures have several advantages. ${ }^{33,35,36}$ Self-reporting may be considered the most practical method of measuring adherence in clinical settings. They also provide a reasonably accurate estimate of adherence. Other advantages include low cost, ease of administration, and flexibility in timing and mode of administration. Also, self-reports can explain underlying issues that contribute to non-adherence. However, self-reported measures of adherence have several potential disadvantages. ${ }^{33,35,36}$ First, many self-reporting measures have not been rigorously tested for convergent or criterion validity, internal consistency, and test-retest reliability. Second, there is a chance of social desirability and memory biases. Patients could have reported themselves as being more adherent than they actually were, and non-adherence may have been underreported due to memory deficit and poor patient recall of information. Here, one of the authors who interviewed patients to obtain self-report adherence data also delivered the adherence-enhancing intervention. This might have increased socially desirable responses. ${ }^{35}$ Third, when using self-reporting measures to assess the efficacy of adherence-enhancing interventions, intervention participants may be disproportionately influenced to self-report faithful adherence compared to control arm participants. ${ }^{35}$

\section{Conclusion}

A pharmacist-led educational intervention had a positive impact on medication adherence in epilepsy patients. This study paves the way for conducting a randomized controlled trial with longer follow-up and more rigorous protocols for measurement of medication adherence to confirm the findings.

\section{Acknowledgments}

Use of the CMMAS is protected by US and International copyright laws. Permission for use is required. A license agreement is available from Donald E. Morisky, MMAS Research (MORISKY) 16636 159th Place SE, Renton WA 98058,dmorisky@gmail.com. This research project was supported by a grant from the Research Center of the Female Scientific and Medical Colleges, Deanship of Scientific Research, King Saud University, Riyadh, Kingdom of Saudi Arabia.

\section{Disclosure}

The authors report no conflicts of interest in this work. 


\section{References}

1. Fisher RS, Van Emde Boas W, Blume W, et al. Epileptic seizures and epilepsy: definitions proposed by the International League Against Epilepsy (ILAE) and the International Bureau for Epilepsy (IBE). Epilepsia. 2005;46(4):470-472.

2. Ngugi AK, Kariuki SM, Bottomley C, Kleinschmidt I, Sander JW, Newton CR. Incidence of epilepsy: a systematic review and metaanalysis. Neurology. 2011;77(10):1005-1012.

3. Al Rajeh S, Awada A, Bademosi O, Ogunniyi A. The prevalence of epilepsy and other seizure disorders in an Arab population: a communitybased study. Seizure. 2001;10(6):410-414.

4. Sabaté E, editor. Adherence to Long-Term Therapies: Evidence for Action. Genava: World Health Organization; 2003.

5. Vrijens B, De Geest S, Hughes DA, et al. A new taxonomy for describing and defining adherence to medications. Br J Clin Pharmacol. 2012; 73(5):691-705.

6. Molugulu N, Gubbiyappa KS, Vasudeva Murthy CR, Lumae L, Mruthyunjaya AT. Evaluation of self-reported medication adherence and its associated factors among epilepsy patients in Hospital Kuala Lumpur. J Basic Clin Pharm. 2016;7(4):105-109.

7. Ferrari CM, de Sousa RM, Castro LH. Factors associated with treatment non-adherence in patients with epilepsy in Brazil. Seizure. 2013; 22(5):384-389.

8. Paschal AM, Rush SE, Sadler T. Factors associated with medication adherence in patients with epilepsy and recommendations for improvement. Epilepsy Behav. 2014;31:346-350.

9. Faught E, Duh MS, Weiner JR, Guérin A, Cunnington MC. Nonadherence to antiepileptic drugs and increased mortality: Findings from the RANSOM Study. Neurology. 2008;71(20):1572-1578.

10. Faught RE, Weiner JR, Guérin A, Cunnington MC, Duh MS. Impact of nonadherence to antiepileptic drugs on health care utilization and costs: findings from the RANSOM study. Epilepsia. 2009;50(3):501-509.

11. Lie IA, Hoggen I, Samsonsen C, Brodtkorb E. Treatment non-adherence as a trigger for status epilepticus: an observational, retrospective study based on therapeutic drug monitoring. Epilepsy Res. 2015;113:28-33.

12. Al-Aqeel S, Gershuni O, Al-Sabhan J, Hiligsmann M. Strategies for improving adherence to antiepileptic drug treatment in people with epilepsy. Cochrane Database Syst Rev. 2017;2:CD008312.

13. Touchette DR, Shapiro NL. Medication compliance, adherence, and persistence: current status of behavioral and educational interventions to improve outcomes. J Manag Care Pharm. 2008;14(6 Suppl D):2-10.

14. Couldridge L, Kendall S, March A. 'A systematic overview - a decade of research'. The information and counselling needs of people with epilepsy. Seizure. 2001;10(8):605-614.

15. Long L, Reeves AL, Moore JL, Roach J, Pickering CT. An assessment of epilepsy patients' knowledge of their disorder. Epilepsia. 2000; 41(6):727-731.

16. Al-Jumah KA, Qureshi NA. Impact of pharmacist interventions on patients' adherence to antidepressants and patient-reported outcomes: a systematic review. Patient Prefer Adherence. 2012;6:87-100.

17. Omran D, Guirguis LM, Simpson SH. Systematic review of pharmacist interventions to improve adherence to oral antidiabetic medications in people with type 2 diabetes. Can J Diabetes. 2012;36(5):292-299.

18. Reis TM, Campos MS, Nagai MM, Pereira LR. Contributions of pharmacists in the treatment of epilepsy: a systematic review. Am J Pharm Benefit. 2016;8(3):e55-e60.

Patient Preference and Adherence

\section{Publish your work in this journal}

Patient Preference and Adherence is an international, peer-reviewed, open access journal that focuses on the growing importance of patient preference and adherence throughout the therapeutic continuum. Patient satisfaction, acceptability, quality of life, compliance, persistence and their role in developing new therapeutic modalities and compounds to optimize
19. No authors listed. ASHP guidelines on pharmacist-conducted patient education and counseling. Am J Health Syst Pharm. 1997;54(4): 431-434.

20. Morisky DE, Ang A, Krousel-Wood M, Ward HJ. Predictive validity of a medication adherence measure in an outpatient setting. $J$ Clin Hypertens (Greenwich). 2008;10(5):348-354.

21. Morisky DE, DiMatteo MR. Improving the measurement of self-reported medication nonadherence: response to authors. $J$ Clin Epidemiol. 2011;64(3):255-257.

22. Krousel-Wood M, Islam T, Webber LS, Re RN, Morisky DE, Muntner P. New medication adherence scale versus pharmacy fill rates in seniors with hypertension. Am J Manag Care. 2009;15(1):59-66.

23. Dash D, Sebastian TM, Aggarwal M, Tripathi M. Impact of health education on drug adherence and self-care in people with epilepsy with low education. Epilepsy Behav. 2015;44:213-217.

24. DiIorio C, Bamps Y, Walker ER, Escoffery C. Results of a research study evaluating WebEase, an online epilepsy self-management program. Epilepsy Behav. 2011;22(3):469-474.

25. Sweileh WM, Ihbesheh MS, Jarar IS, et al. Self-reported medication adherence and treatment satisfaction in patients with epilepsy. Epilepsy Behav. 2011;21(3):301-305.

26. Li J, Si Y, Hu J, et al. Enhancing medical compliance of patients with convulsive epilepsy in rural community: a randomized intervention trial. Epilepsia. 2013;54(11):1988-1996.

27. Peterson GM, McLean S, Millingen KS. A randomised trial of strategies to improve patient compliance with anticonvulsant therapy. Epilepsia. 1984;25(4):412-417.

28. Ibinda F, Mbuba CK, Kariuki SM, et al. Evaluation of Kilifi epilepsy education programme: a randomized controlled trial. Epilepsia. 2014; 55(2):344-352.

29. Tang F, Zhu G, Jiao Z, Ma C, Chen N, Wang B. The effects of medication education and behavioral intervention on Chinese patients with epilepsy. Epilepsy Behav. 2014;37:157-164.

30. Fogg A, Staufenberg EF, Small I, Bhattacharya D. An exploratory study of primary care pharmacist-led epilepsy consultations. Int $J$ Pharm Pract. 2012;20(5):294-302.

31. Plumpton CO, Brown I, Reuber M, Marson AG, Hughes DA. Economic evaluation of a behavior-modifying intervention to enhance antiepileptic drug adherence. Epilepsy Behav. 2015;45:180-186

32. Simon-Tuval T, Neumann PJ, Greenberg D. Cost-effectiveness of adherence-enhancing interventions: a systematic review. Expert Rev Pharmacoecon Outcomes Res. 2016;16(1):67-84.

33. Garfield S, Clifford S, Eliasson L, Barber N, Willson A. Suitability of measures of self-reported medication adherence for routine clinical use: a systematic review. BMC Med Res Methodol. 2011;11:149.

34. Costa E, Giardini A, Savin M, et al. Interventional tools to improve medication adherence: review of literature. Patient Prefer Adherence. 2015; 9:1303-1314.

35. Stirratt MJ, Dunbar-Jacob J, Crane HM, et al. Self-report measures of medication adherence behavior: recommendations on optimal use. Transl Behav Med. 2015;5(4):470-482.

36. Voils CI, Hoyle RH, Thorpe CT, Maciejewski ML, Yancy WS Jr. Improving the measurement of self-reported medication nonadherence. J Clin Epidemiol. 2011;64(3):250-254. clinical outcomes for existing disease states are major areas of interest for the journal. This journal has been accepted for indexing on PubMed Central. The manuscript management system is completely online and includes a very quick and fair peer-review system, which is all easy to use. Visit http://www. ovepress.com/testimonials.php to read real quotes from published authors. 